Kanazawa-94-09

KITP-94-02

May, 1994

\title{
Minimal String Unification and Constraint on Hidden Sector
}

\author{
Hiroshi Kawabe, Tatsuo Kobayashi* ${ }^{*}$ and Noriyasu Ohtsubo ${ }^{* *}$ 目 \\ Yonago National College of Technology, \\ Yonago, 683, Japan \\ *Department of Physics, Kanazawa University, \\ Kanazawa, 920-11, Japan \\ **Kanazawa Institute of Technology, \\ Ishikawa 921, Japan
}

\begin{abstract}
We examine whether a minimal string model possessing the same massless spectra as the MSSM can be obtained from $Z_{4}, Z_{6}$ and $Z_{8}$ orbifold constructions. Using an anomaly cancellation condition of the target space duality symmetry, we derive allowable values of a level $k_{1}$ of $\mathrm{U}(1)_{Y}$ for the minimal string model on the orbifolds through computer analyses. We investigate threshold corrections of the gauge coupling constants of $\mathrm{SU}(3)$, $\mathrm{SU}(2)$ and $\mathrm{U}(1)_{Y}$ and examine consistencies of the model with the LEP experiments. It is found that $Z_{4}$ and $Z_{8}$-II can not derive the minimal string model but $Z_{6}$-I, $Z_{6}$-II and $Z_{8}$-I are possible to derive it with $k_{1}=29 / 21$, $1 \leq k_{1} \leq 32 / 21$ and $1 \leq k_{1} \leq 41 / 21$ respectively. We obtain explicitly allowed combinations of modular weights. The minimum values of the moduli on unrotated planes are estimated within the ranges of the levels. Further we investigate what kinds of hidden sectors are consistent with the minimal string models. Also their gauge coupling constants of the hidden groups are estimated. We discuss Yukawa couplings of the models.
\end{abstract}

\footnotetext{
${ }^{\dagger}$ e-mail:kobayasi@hep.s.kanazawa-u.ac.jp

‡e-mail:ohtsubo@neptune.cisp.kanazawa-it.ac.jp
} 


\section{Introduction}

Superstring theories are the only known candidates for unified theories of all the interactions including gravity. All the gauge coupling constants are unified even without a unified group at a string scale $M_{\mathrm{st}}=5.27 \times g_{\mathrm{st}} \times 10^{17} \mathrm{GeV}$ [1], where $g_{\mathrm{st}} \simeq 1 / \sqrt{2}$ is a universal string coupling constant. There are crucial problems how to derive the minimal supersymmetric standard model (MSSM) at the low energy from superstring theories and how to break the supersymmetry (SUSY). For the first problem, several types of scenarios have been discussed to lead to the MSSM spectrum. Some of them have intermediate scales of grand unified theories (GUTs) like SU(5) or SO(10), and others have extra matter fields other than the MSSM spectrum. However GUTs often face problems on triplet-doublet splitting in the Higgs sector in addition to mass spliting of the quarks and leptons other than the third generation. Further some models with extra matter fields lead to the fast proton decay. Here we concentrate ourselves to the simplest scenario that the massless spectrum at $M_{\mathrm{st}}$ is same as one of the MSSM. That does not suffer the above problems and is called a minimal string model. Explicit searches for the model have been done, e.g., within the framework of orbifold models.

The construction of the 4-dim string vacua through the orbifolds is one of the simplest methods among several types of constructions [2]. For the orbifold models, we can obtain several phenomenological aspects such as Yukawa couplings, Kähler potentials, threshold corrections of the gauge coupling constants and so on. Although some standard-like models have been found in the orbifold models [3], a completely realistic model has never been derived. It seems that needed are phenomenological constraints to obtain the realistic model from a huge number of 4-dim string vacua.

Recent study of the LEP measurements shows that all the gauge couplings of the MSSM are unified at $M_{X} \sim 10^{16} \mathrm{GeV}$ 进. A difference between $M_{X}$ and $M_{\text {st }}$ seems to reject the possibility for the minimal string model. However it is expected that this difference is explained by threshold corrections of the gauge couplings due to higher massive modes of the string. The corrections have been calculated in the orbifold models [5, 6]. The corrections as well as masses depend on moduli $T$, whose vacuum expectation values describe orbifold geometries. Large values of the moduli lead to large threshold corrections, although a fairly 
larger moduli value seems unnatural. Refs. [7, 8] studied the threshold corrections consistent with the measured values of the gauge couplings at $M_{Z}$ in the case where a Kac-Moody level $k_{1}$ of $U(1)_{Y}$ is equal to $5 / 3$. This value of $k_{1}$ is predicted by GUTs, but string theories could derive any other level [9]. Thus, it is very interestring to extend to the cases of general levels. In ref. [10], the levels of $Z_{N} \times Z_{M}$ orbifold models are estimated as $1 \leq k_{1} \lesssim 2$ in order to explain the measured values of the gauge coupling constants at $M_{Z}$. In this paper we study in detail the possibility for obtaining the minimal string model from $Z_{N}$ orbifolds in the case where $1 \leq k_{1}<2$.

In the above discussion a target-space duality symmetry [11] is very important and that is a "stringy" feature. Also an effective lagrangian is invariant under the symmetry of the moduli fields. However loop effects due to only massless modes make the symmetry anomalous [6]. The duality anomaly can be cancelled by two ways. One is the Green-Schwarz mechanism [12, 13, 6], which induces a nontrivial transformation of a dilaton field $S$ under the duality transformation. Further the anomaly can be cancelled in terms of the moduli dependent threshold corrections of the gauge couplings due to the massive modes. The former is independent of gauge groups and it constrains strongly the massless spectrum, such as gauge anomalies constrains spectra in field theories. . The strong constraint makes possible to investigate massless spectra instead of the explicit search by shifts and Wilson lines [14, 15, 16] on an $\mathrm{E}_{8} \times \mathrm{E}_{8}^{\prime}$ lattice. The anomaly cancellation condition forbids the minimal string model in $Z_{3}$ and $Z_{7}$ orbifild models [8]. We do not study here the $Z_{3}$ and $Z_{7}$ orbifold models. Further we omit the $Z_{12}$ orbifold models, which have several types of twisted sectors. Thus the $Z_{12}$ orbifold models need longer analysis. However we can study the $Z_{12}$ orbifold models in a way similar to the following discussion.

Hidden sectors play a role in the SUSY-breaking. A gaugino condensation in the hidden sector is one of the realistic SUSY-breaking mechanisms [17], which determines vacuum expectation values of the dilaton and moduli fields [18]. The expectation value of $S$ derives a gauge coupling constant. Refs. [18] show that a scalar potential of the moduli fields has a minimum around a self-dual point of the duality. Also effective one-loop potential is considered in refs. [19]. The gaugino condensation around $10^{13} \mathrm{GeV}$ could lead to soft SUSY-breaking terms

* Note that the $Z_{N} \times Z_{M}$ orbifold models do not have such a constraint, because all the moduli of the three planes contribute the threshold corrections. 
at the weak scale. The duality anomaly cancellation condition restricts the hidden sector so as to be consistent with the MSSM as discussed in ref.[20], where the duality anomaly cancellation condition on $\mathrm{U}(1)_{Y}$ was not taken into account. In this paper we study the allowed gauge groups and matter fields in hidden sectors of the minimal string models derived from the $Z_{6}$-I, $Z_{6}$-II and $Z_{8}$-II orbifolds. The consistency with $\mathrm{U}(1)_{Y}$ is investigated in more detail. Also estimated are their gauge coupling constants at $10^{13} \mathrm{GeV}$ and their blow-up scales.

This paper is organized as follows. In section two we review on the orbifold models. Also their massless conditions are studied to find conditions on oscillation numbers of the MSSM matter fields under some values of the level $k_{1}$. In section three we review on the duality symmetry and the threshold corrections. The discussion of section two leads to allowed modular weights for each MSSM matter field. The duality anomaly cancellation condition is also reviewed. In section four we investigate the possibility to derive the minimal string model with consistent values of the measured gauge couplings from the $Z_{N}$ orbifold models. We try to assign the allowed modular weights to matter fields of the MSSM and find combinations of the modular weights to satisfy the duality anomaly cancellation condition for the $\mathrm{SU}(3), \mathrm{SU}(2)$ and $\mathrm{U}(1)_{Y}$ gauge groups. Through such an analysis we get allowed values of the level $k_{1}$. Then we study whether the allowed combinations lead to the threshold corrections consistent with the measurements. Some tables show explicitly the combinations, which are useful for model building. Here we restrict ourselves to the case where the vacuum expectation values of the muduli fields are of order one. In section five we find the hidden sectors which are consistent with the observable massless spectra obtained in section four from viewpoint of the duality anomaly cancellation conditions. That does not allow hidden sectors which have smaller number of matter fields with non-trivial representations under hidden gauge groups. Then we estimate their hidden gauge coupling constants at $10^{13} \mathrm{GeV}$ and their blow-up scales. In section six we study Yukawa couplings of the allowed models, explicitly. That could also be used for phenomenological constraints. Section seven is devoted to conclusions and discussions.

\section{2. $Z_{N}$ Orbifold Models}


In the orbifold models [2], the string states consist of the bosonic strings on the 4-dim space-time and a 6-dim orbifold, their right-moving superpartners and left-moving gauge parts whose momenta span a shifted $\mathrm{E}_{8} \times \mathrm{E}_{8}^{\prime}$ lattice. The right-moving fermionic parts are bosonized and momenta of the bosonized fields span an SO(10) lattice. The 6-dim orbifolds are obtained through the division of a 6 -dim space $\mathrm{R}^{6}$ by 6 -dim Lie lattices and their automorphisms (twists). We use here an $\mathrm{SO}(5)^{2} \times \mathrm{SU}(2)^{2}$ lattice for the $Z_{4}$ orbifold, a $\mathrm{G}_{2}^{2} \times \mathrm{SU}(3)$ lattice for the $Z_{6}$-I, a $\mathrm{G}_{2} \times \mathrm{SU}(3) \times \mathrm{SU}(2)^{2}$ lattice for the $Z_{6}$-II, an $\mathrm{SO}(9) \times \mathrm{SO}(5)$ lattice for the $Z_{8}$ - I and an $\mathrm{SO}(9) \times \mathrm{SU}(2)^{2}$ lattice for the $Z_{8}$-II [16]. Using other lattice, we can construct these orbifolds. For any lattice, we can study in a way similar to the following discussion. We denote eigenvalues of the twist $\theta$ in a complex basis $\left(X_{i}, \tilde{X}_{i}\right)(i=1,2,3)$ as $\exp \left[2 \pi i v^{i}\right]$, whose exponents $v^{i}$ are $(1,1,2) / 4$ for the $Z_{4}$ orbifold, $(1,1,4) / 6$ for the $Z_{6}$-I, $(1,2,3) / 6$ for $Z_{6}$-II, $(1,5,2) / 8$ for the $Z_{8}$-I and $(1,3,4) / 8$ for the $Z_{8}$-II. The twist $\theta$ is embedded into the $\mathrm{SO}(10)$ and $\mathrm{E}_{8} \times \mathrm{E}_{8}^{\prime}$ lattices in terms of shifts so that the $N=1$ SUSY remains and the gauge group breaks into a small one. The $\mathrm{E}_{8} \times \mathrm{E}_{8}^{\prime}$ lattice is shifted by Wilson lines, as well.

There are two types of closed strings on the orbifolds. One is an untwisted string whose massless states should satisfy

$$
h-1=0
$$

where $h$ is a conformal dimension of the $\mathrm{E}_{8} \times \mathrm{E}_{8}^{\prime}$ gauge part. The other is a twisted string. Massless states of $\theta^{\ell}$-twisted sector should satisfy the following condition:

$$
h+N_{i}+c_{\ell}-1=0,
$$

where $N_{i}$ is an oscillation number associated with the $i$-th plane and $c_{\ell}$ is obtained from

$$
c_{\ell}=\frac{1}{2} \sum_{i=1}^{3} v_{\ell}^{i}\left(1-v_{\ell}^{i}\right), \quad v_{\ell}^{i} \equiv \ell v^{i}-\operatorname{Int}\left(\ell v^{i}\right) .
$$

Here Int $(a)$ represents an integer part of $a$.

A representation $\underline{R}$ of the non-abelian group $G$ contributes to the conformal dimension as

$$
h=\frac{C(\underline{R})}{C(G)+k}
$$

where $k$ is a level of a Kac-Moody algebra and $C(G)(C(\underline{R}))$ is a quadratic Casimir of the adjoint $(\underline{R})$ representation of the group $G$, e.g., $C(\operatorname{SU}(N))=N$ 
$\left(C(\underline{N})=\left(N^{2}-1\right) / 2 N\right), C(\mathrm{SO}(2 n))=2 n-2(C(\underline{2 n})=(2 n-1) / 2), C\left(\mathrm{E}_{6}\right)=12$ $(C(\underline{27})=26 / 3), C\left(\mathrm{E}_{7}\right)=18(C(\underline{56})=57 / 4)$ and $C\left(\mathrm{E}_{8}\right)=30$. In general the string theories derive the gauge groups with $k=1$, I except for $\mathrm{U}(1)$. Then we restrict ourselves to the case where $k=1$ for the non-abelian groups. It follows that the representations $\underline{N}$ of $\mathrm{SU}(N), \underline{2 n}$ (vector) of $\mathrm{SO}(2 n), \underline{27}$ of $\mathrm{E}_{6}$ and $\underline{56}$ of $\mathrm{E}_{7}$ group have the conformal dimensions of $h=(N-1) / 2 N, 1 / 2,2 / 3$ and $3 / 4$, respectively. A state with a charge $Q$ of the $\mathrm{U}(1)_{Y}$ has an additional contribution of $h=Q^{2} / k_{1}$ where $k_{1}$ is the level of $\mathrm{U}(1)_{Y}$.

It is found that all the above representations are possible to satisfy the massless condition (2.1) in the untwisted sector if they have suitable charges of the extra U(1)'s. However, they are not always satisfy the massless condition (2.2) in the twisted sector. We can get higher bounds of the oscillation number $N_{i}$ for each representations from the condition (2.2). For the MSSM matter fields, the bounds depend on the level $k_{1}$. For example we consider a quark singlet with $\mathrm{U}(1)_{Y}$ charge $Q$. For this state to have $N_{i}$, the level $k_{1}$ should satisfy the following bound:

$$
k_{1} \geq \frac{Q^{2}}{2 / 3-c_{\ell}-N_{i}} .
$$

For the other MSSM matter fields, we have similar relations. Existence of each MSSM matter field in the untwisted sector gives a lower bound of $k_{1}$. We have $k_{1} \geq 1$ so that singlets with $Q=1$ appear.

\section{Duality and Threshold Corrections}

The duality symmetry is retained in effective field theories derived from the orbifold models [22]. In the theories, moduli fields $T_{i}(i=1,2,3)$ associated with the $i$-th complex planes have the Kähler potentials

$$
-\sum_{i} \log \left|T_{i}+\bar{T}_{i}\right|
$$

which are invariant under a duality transformation:

$$
T_{i} \rightarrow \frac{a_{i} T_{i}-i b_{i}}{i c_{i} T_{i}+d_{i}}
$$

up to the Kähler transformation, where $a_{i}, b_{i}, c_{i}, d_{i} \in \mathbf{Z}$ and $a_{i} d_{i}-b_{i} c_{i}=1$.

\footnotetext{
${ }^{\dagger}$ Gauge groups with $k \neq 1$ are discussed in refs. 21.
} 
The Kähler potential of the matter field $A$ is

$$
\prod_{i=1}^{3}\left(T_{i}+\bar{T}_{i}\right)^{n^{i}} A \bar{A}
$$

whose duality invariance requires the following transformation:

$$
A \rightarrow A \prod_{i=1}^{3}\left(i c_{i} T_{i}+d_{i}\right)^{n^{i}}
$$

where $n^{i}$ is called a modular weight [23, 8].

For the untwisted sector associated with the $p$-th plane, the matter fields have $n^{i}=-\delta_{p}^{i}$. The $\theta^{\ell}$-twisted state without oscillators has the following modular weights:

$$
\begin{array}{ll}
n^{i}=v_{\ell}^{i}-1, & v_{\ell}^{i} \neq 0, \\
n^{i}=0, & v_{\ell}^{i}=0 .
\end{array}
$$

The oscillator $\partial X_{i}$ reduces the corresponding element of the modular weight by one and the oscillator $\partial \tilde{X}_{i}$ contributes oppositely. Thus we can obtain modular weights of the matter fields using the allowable values for $N_{i}$.

The allowed modular weights for the MSSM matter fields depend on the value of the level $k_{1}$. Table 1 lists the modular weights and the lower bounds of $k_{1}$ for each MSSM matter field permitted by the massless condition in the previous section for $\theta$ - and $\theta^{2}$-twisted sectors in the $Z_{4}$ orbifold model. (In the table $\theta^{3}$ twisted sector is omitted because it includes only anti-matters [24. Such sectors are also omitted in the following tables.) In the table, the underline represents any permutation of the elements. For example, the representations $(3,2)_{1 / 6}$, $(\overline{3}, 1)_{1 / 3},(\overline{3}, 1)_{-2 / 3},(1,2)_{ \pm 1 / 2}$ and $(1,1)_{1}$ in $\theta$ twisted sector are able to possess $n^{i}=(-3,-3,-2) / 4$, if $k_{1} \geq 4 / 15,16 / 51,64 / 51,4 / 7$ and 16/11 respectively. The modular weight $n^{i}=(\underline{-7,-3},-2) / 4$ is not realized for $(3,2)_{1 / 6},(\overline{3}, 1)_{-2 / 3}$ and $(1,1)_{1}$ in the case where $k_{1}<2$. It seems that the orbifold models compatible with the experiments derive $k_{1}<2$ as suggested in the previous study about the $Z_{N} \times Z_{M}$ orbifolds [10]. Then we limit the subsequent studies to the cases of $k_{1}<2$. For the twisted sectors of $Z_{6}$-I, $Z_{6}$-II, $Z_{8}$-I and $Z_{8}$-II, the modular weights and the lower bounds of $k_{1}$ for the MSSM matter fields are listed in Table 2, Table 3, Table 4 and Table 5, respectively, where we omit the modular weights requiring $k_{1} \geq 2$. The $Z_{12}$ orbifold models have much more modular weights than the others. That makes analysis on the $Z_{12}$ orbifolds lengthy. However, we can obtain modular weights of the $Z_{12}$ orbifolds, similarly. 
When we consider the hidden sectors, we do not have to take care of the level $k_{1}$ of $\mathrm{U}(1)_{Y}$. The last column of Table 2 shows which of modular weights can be possessed by the hidden matter fields with the $N$-dim representation of the $Z_{6}$-I orbifold models. Plus signs in the column denote that their representations are permitted by the massless condition (2.2) for all $\mathrm{SU}(N)^{\prime}$. Bounds of $N$ are given for the representation $\underline{N}$ of $\mathrm{SU}(N)^{\prime}$. We remark that the $Z_{8}$ orbifold models are possible to have $N \leq 8$ in $\mathrm{SU}(N)$, while the $Z_{6}$ models are $N \leq 9$ in $\mathrm{SU}(N)$ by the explicit search of gauge groups in terms of shifts and Wilson lines [25, 26]. In addition to the modular weights in Table 2 , the matter fields with the $N$-dim representation of $\mathrm{SU}(N)^{\prime}(N \leq 3)$ are allowed to have $n^{i}=(\underline{-10,-4,-4}) / 6$, and doublets of $\mathrm{SU}(2)^{\prime}$ are able to possess the following modular weights,

$$
\begin{gathered}
\underline{(-23,-5},-2) / 6, \quad \underline{(-17,-11},-2) / 6, \quad \underline{(-11,-5}, 4) / 6, \\
(\underline{-9,-3}, 0) / 6, \quad(\underline{3,-3}, 0) / 6 .
\end{gathered}
$$

Similarly the last column of Table 3 shows the allowed hidden matter fields with the modular weights. In addition, the matter fields with the $N$-dim representation of $\mathrm{SU}(N)^{\prime}(N \leq 3)$ are possible to have $n^{i}=(-17,-4,-3) / 6$ and $(-5,-10,-3) / 6$, and further doublets of $\mathrm{SU}(2)^{\prime}$ are able to possess $n^{i}=$ $(\underline{-9}, 0, \underline{-3}) / 6$ and $(\underline{3}, 0, \underline{-3}) / 6$. For the $Z_{8}$-I orbifold models, all matter fields with the $N$-dim representations of $\mathrm{SU}(N)^{\prime}$ are allowed to have every modular weight shown in Table 4 . In addition, the matter fields with the $N$-dim representation of $\mathrm{SU}(N)^{\prime}(N \leq 3)$ are possible to have the following modular weights,

$$
(\underline{-31,-3},-6) / 8, \quad \underline{(-15,-3},-14) / 8, \quad \underline{(-7,5},-6) / 8 .
$$

Furthermore doublets of $\mathrm{SU}(2)^{\prime}$ are able to possess $\left.n^{i}=\underline{(-12,-4}, 0\right) / 8$ and $(\underline{4,-4}, 0) / 8$.

The duality symmetry becomes anomalous by loop effects of only massless modes [6]. Triangle diagrams contributing to the duality anomaly have two gauge bosons and moduli dependent connections like Kähler or $\sigma$-model connections as external lines, and massless fermions in addition to gauginos as internal lines. Duality anomaly coefficients $b^{i}$ of a group $G$ are obtained from

$$
b^{i}=-C(G)+\sum_{\underline{R}} T(\underline{R})\left(1+2 n_{\underline{R}}^{i}\right),
$$

where $T(\underline{R})$ is an index given by $T(\underline{R})=C(\underline{R}) \operatorname{dim}(\underline{R}) / \operatorname{dim}(G)$, e.g., $T(\underline{R})=1 / 2$ for the $N$-dim fundamental representation of $\mathrm{SU}(N)$. 
The duality anomaly can be cancelled by two ways. One is the Green-Schwarz (GS) mechanism [12], which induces a non-trivial transformation to the dilaton field $S$ under the duality as

$$
S \rightarrow S-\frac{1}{8 \pi^{2}} \sum_{i=1}^{3} \delta_{\mathrm{GS}}^{i} \log \left(i c_{i} T_{i}+d_{i}\right),
$$

where $\delta_{\mathrm{GS}}^{i}$ is a GS coefficient. Note that the above mechanism is independent of gauge groups. Further the duality anomaly can be cancelled in terms of moduli dependent threshold corrections due to massive modes. The corrections depend only on the moduli whose planes are unrotated under some twist, because only $N=2$ sectors contribute to the moduli dependent threshold corrections of the gauge coupling constants. For the other planes, the duality anomaly should be cancelled only by the GS mechanism. It works, if the following condition is satisfied:

$$
b_{3}^{\prime j}=b_{2}^{\prime j}=b_{1}^{\prime j} / k_{1}=b_{\text {hid }}^{\prime j},
$$

for $j$-th planes rotated under any twist, where $b_{3}^{\prime j}, b_{2}^{\prime j}$ and $b_{1}^{\prime j}$ are the anomaly coefficients of $\mathrm{SU}(3), \mathrm{SU}(2)$ and $\mathrm{U}(1)_{Y}$, respectively, for the minimal string model in the observed sector, as discussed in ref. [8]. The anomaly coefficient in the hidden sector is represented by $b_{\text {hid }}^{\prime j}$. Only the first plane is concerned with eq. (3.8) in the $Z_{6}$-II orbifold models, whereas the first and the second planes are in $Z_{4}, Z_{6}$-I, $Z_{8}$-I and $Z_{8}$-II orbifold models. The equation (3.8) gives more stringent constraints to the latter orbifold models because $k_{1}$ is common for the both planes.

The other $k$-th $(k \neq j)$ planes contribute in the threshold corrections of the gauge coupling constants induced by the tower of higher massive modes. The threshold corrections [5, 6] are given by

$$
\Delta_{a}\left(T_{k}\right)=-\frac{1}{16 \pi^{2}} \sum_{k}\left(b_{a}^{\prime k}-k_{a} \delta_{\mathrm{GS}}^{k}\right) \log \left|\eta\left(T_{k}\right)\right|^{4},
$$

where $\eta(T)=e^{-\pi T / 12} \prod_{n \geq 1}\left(1-e^{-2 \pi n T}\right)$ is the Dedekind function. Eq. (3.9) may be modified in the cases of the orbifolds constructed through other lattices said above [27]. Also presence of non-vanishing Wilson lines modify the threshold corrections as well as the duality symmetry [28]. However the Wilson lines vanish for the $N=2$ sector [15, 16]. Thus we do not need the modification. The moduli $T_{2}$ and $T_{3}$ participate in eq. (3.9) for the $Z_{6}$-II orbifold, while only $T_{3}$ for the other orbifolds. For simplicity, we consider a case of $T_{2}=T_{3}\left(=T_{k}\right)$ in the $Z_{6}$-II orbifold 
models. Then we denote $\delta_{\mathrm{GS}}^{k}=\delta_{\mathrm{GS}}^{2}+\delta_{\mathrm{GS}}^{3}, b_{a}^{\prime k}=b_{a}^{\prime 3}+b_{a}^{\prime 2}$ and $n_{\underline{R}}^{k}=n_{\underline{R}}^{2}+n_{\underline{R}}^{3}$ for the $Z_{6}$-II and remove the summation.

Using the threshold corrections, we obtain the one-loop coupling constants $\alpha_{a}(\mu)=k_{a} g_{a}^{2}(\mu) / 4 \pi\left(k_{3}=k_{2}=k_{\text {hid }}=1\right)$ at an energy scale $\mu$ as follows,

$$
\alpha_{a}^{-1}(\mu)=\alpha_{\mathrm{st}}^{-1}+\frac{1}{4 \pi} \frac{b_{a}}{k_{a}} \log \frac{M_{\mathrm{st}}^{2}}{\mu^{2}}-\frac{1}{4 \pi}\left(\frac{b_{a}^{\prime k}}{k_{a}}-\delta_{\mathrm{GS}}^{k}\right) \log \left[\left(T_{k}+\bar{T}_{k}\right)\left|\eta\left(T_{k}\right)\right|^{4}\right],
$$

where $\alpha_{\text {st }}=g_{\mathrm{st}}^{2} / 4 \pi$ and $b_{a}$ are $N=1 \beta$-function coefficients. We use the same $b_{3}=-3, b_{2}=1$ and $b_{1}=11$ as ones of the MSSM. There are also moduliindependent threshold corrections [1]. It is expected that moduli-independent corrections are smaller than moduli-dependent one. In this paper we neglect moduli-independent corrections, although the neglect leads to small uncertainty.

Here we discuss the unification of $\mathrm{SU}(3)$ and $\mathrm{SU}(2)$ gauge couplings. The renormalization group equation (3.10) relates the unification scale $M_{X}$ of SU(3) and $\mathrm{SU}(2)$ with $M_{\mathrm{st}}$ as follows [8],

$$
\log \frac{M_{X}}{M_{\mathrm{st}}}=\frac{1}{8} \Delta b^{\prime k} \log \left[\left(T_{k}+\bar{T}_{k}\right)\left|\eta\left(T_{k}\right)\right|^{4}\right],
$$

where $\Delta b^{\prime k} \equiv b_{3}^{\prime k}-b_{2}^{\prime k}$. Note that the $\mathrm{U}(1)_{Y}$ gauge coupling does not necessarily unify with $\mathrm{SU}(3)$ and $\mathrm{SU}(2)$ at $M_{X}$, because $k_{1}$ is not always equal to $k_{1}=5 / 3$. The term $\log \left[\left(T_{k}+\bar{T}_{k}\right)\left|\eta\left(T_{k}\right)\right|^{4}\right]$ is negative for any value of $T$. Thus to obtain $M_{X}<M_{\text {st }}$, the anomaly coefficients should satisfy $\Delta b^{\prime k}>0$. Assuming the SUSY is broken at $M_{Z}$, we have

$$
\log \frac{M_{X}^{2}}{M_{Z}^{2}}=\pi\left(\sin ^{2} \theta_{W}\left(M_{Z}\right) \alpha_{\mathrm{em}}^{-1}\left(M_{Z}\right)-\alpha_{3}^{-1}\left(M_{Z}\right)\right) .
$$

Then we use $\sin ^{2} \theta_{W}\left(M_{Z}\right)=0.2325 \pm .0008, \alpha_{\mathrm{em}}^{-1}\left(M_{Z}\right)=127.9 \pm .1, \alpha_{3}^{-1}\left(M_{Z}\right)=$ $8.82 \pm .27$ at $M_{Z}=91.173 \pm .020$ to obtain

$$
M_{X}=10^{16.23 \pm .27} \mathrm{GeV}, \quad \alpha_{X}^{-1}=24.51 \pm .09,
$$

where $\alpha_{X} \equiv \alpha_{3}\left(M_{X}\right)=\alpha_{2}\left(M_{X}\right)$. In the case where $\Delta b^{\prime k}=3$, we obtain $T \simeq 11$ through (3.11) using $M_{X}=10^{16.2} \mathrm{GeV}$ and $M_{\text {st }}=10^{17.6} \mathrm{GeV}$.

We eliminate $\alpha_{\mathrm{st}}, \delta_{\mathrm{GS}}^{k}$ and $T_{k}$ in $(3.10)$ for $\alpha_{3}^{-1}, \alpha_{2}^{-1}$ and $\alpha_{1}^{-1}$ to obtain

$$
\begin{aligned}
\left(k_{1} b_{2}^{\prime k}-b_{1}^{\prime k}\right) \alpha_{3}^{-1}(\mu) & =\left(b_{2}^{\prime k}-b_{3}^{\prime k}\right) \alpha_{\mathrm{em}}^{-1}(\mu)-\left\{b_{1}^{\prime k}+b_{2}^{\prime k}-\left(k_{1}+1\right) b_{3}^{\prime k}\right\} \alpha_{\mathrm{em}}^{-1}(\mu) \sin ^{2} \theta_{\mathrm{W}}(\mu) \\
& +\frac{1}{4 \pi}\left\{4 b_{1}^{\prime k}-\left(3 k_{1}+11\right) b_{2}^{\prime k}-\left(k_{1}-11\right) b_{3}^{\prime k}\right\} \log \frac{M_{\mathrm{st}}^{2}}{\mu^{2}}
\end{aligned}
$$




\section{Minimal String Model}

In this section we study the possibility for the minimal string model, using the $Z_{N}$ orbifold models. For the purpose, we use the duality anomaly cancellation condition instead of searching explicitly massless spectra in terms of all the possible shifts and the Wilson lines on the $\mathrm{E}_{8} \times \mathrm{E}_{8}^{\prime}$ lattice. First of all, we assign allowed modular weights to the MSSM matter fields, i.e., the three $(3,2)_{1 / 6}$ representations, three $(\overline{3}, 1)_{-2 / 3}$, three $(\overline{3}, 1)_{1 / 3}$, five $(1,2)_{ \pm 1 / 2}$ and three $(1,1)_{1}$, to find combinations of the modular weights satisfying the duality anomaly cancellation condition (3.8). Note that the presence or absence of the right-handed neutrinos does not affect the following discussion, because they are singlets with $Q=0$ under the standard gauge group. As shown in ref. [8], we cannot derive any combination satisfying (3.8) from the $Z_{3}$ and $Z_{7}$ orbifold models, whose orbifolds are constructed through $\mathrm{SU}(3)^{3}$ and $\mathrm{SU}(7)$ lattices and have exponents $v^{i}=(1,1,1) / 3$ and $(1,2,4) / 7$, respectively.

For each allowed combination, we obtain the level $k_{1}$ by a ratio of the anomaly coefficients, i.e., $k_{1}=b_{1}^{\prime j} / b_{3}^{\prime j}$. Here we have to check whether or not each combination includes modular weights allowed by this level $k_{1}$.

Next we study the possibility that the threshold corrections due to massive modes derive the measured gauge coupling constants. Namely we investigate whether or not combinations of the modular weights allowed at the above stage derive the gauge coupling constants consistent with all measurements falling within the error bars, through (3.14). Here we estimate $\alpha_{3}^{-1}\left(M_{Z}\right)$ through (3.14) using the value of $k_{1}$ obtained through (3.8) and the measured values of $\sin ^{2} \theta_{W}\left(M_{Z}\right)$ and $\alpha_{\mathrm{em}}\left(M_{Z}\right)$, then compare it with the experimental value $\alpha_{3}^{-1}\left(M_{Z}\right)$ in order to investigate the consistency of the model. Here we restrict ourselves to the case where $T_{k} \leq 11$ and $\Delta b^{\prime k} \geq 3$, in order to investigate the possibility that $T_{k}$ is of order one. However, we can analyze other case in a similar way. The $Z_{4}$ and $Z_{8}$-II orbifold models cannot lead to the minimal string model consistent with the measured gauge coupling constants. For example $Z_{4}$ orbifold models lead $\alpha_{3}^{-1}\left(M_{Z}\right) \leq 4.0$ through (3.14) using the measured values of $\sin ^{2} \theta_{W}\left(M_{Z}\right)$ and

$\ddagger$ Using (3.14) and experimental values of the gauge couplings $\alpha_{a}^{-1}\left(M_{z}\right)(a=1,2,3)$, we can estimate the value of $k_{1}$ through (3.14) [10]. The value $k_{1}$ can be compared with $k_{1}$ obtained by (3.8) to investigate the consistency of the models. This analysis derives the same results as the procedure studied here. 
$\alpha_{\mathrm{em}}\left(M_{Z}\right)$. For the $Z_{6}$-I orbifold models, we have only one combination of modular weights consistent with the measurements in the case where $\Delta b^{\prime k} \geq 3$. Even for the case $0 \leq \Delta b^{\prime k} \leq 3$, we can find only five combinations, which are shown in Table 6. In the table the MSSM matter fields are represented by $Q, U, D, L$, $E$ and $H$ for the quark doublets, the quark singlets of the up-sector and downsector, the lepton doublets, lepton singlets and the Higgs fields, respectively. In the table modular weights for the matter fields are represented by the following numbers:

$$
\begin{gathered}
1:(-1,0,0), \quad 2:(0,-1,0), \quad 3:(0,0,-1), \\
4:(-5,-5,-2) / 6, \quad 5:(-3,-3,0) / 6, \quad 6:(-4,-4,-4) / 6, \\
7:(-11,-5,-2) / 6, \quad 8:(-5,-11,-2) / 6, \quad 9:(-5,-5,4) / 6 .
\end{gathered}
$$

For the $Z_{8}$-I orbifold models, 104 combinations with $\Delta b^{\prime k} \geq 3$ are allowed, including 52 combinations with $\Delta b^{\prime k}=4$, which is the largest value among them and leads to $T_{k} \simeq 8.8$, using $M_{X}=10^{16.2} \mathrm{GeV}$ and $M_{\text {st }}=10^{17.6} \mathrm{GeV}$. These combinations with $\Delta b^{\prime k}=4$ and 3 are classified into 13 and 15 types by values of $b_{3}^{\prime i}$ and $b_{2}^{\prime i}$ as shown in Table 7 , where the values of $b_{1}^{\prime k}$ are omitted. The combinations with $\Delta b^{\prime k}=4$ derive $1 \leq k_{1} \leq 41 / 21$, including $5 / 3$, while the cases with $\Delta b^{\prime k}=3$ do not lead to $5 / 3$.

The $Z_{6}$-II orbifold models allow 4586 combinations, which include a combination with $\Delta b^{\prime k}=6$ as the largest value leading $T_{k}=6.5$. Among the 4586 combinations, 632 ones with $\Delta b^{\prime} \geq 4$ are classified by values of $b_{3}^{\prime i}$ and $b_{2}^{\prime i}$ as shown in Tables 8-1 and 8-2, where the values of $b_{1}^{\prime k}$ are omitted. As shown in the tables, the minimal string model with $k_{1}=5 / 3$ cannot be derived from $Z_{6}$-II orbifold. However, if we permit the cases with $0 \leq \Delta b^{\prime k}<4$, we can have combinations of modular weights leading the measured couplings and $k_{1}=5 / 3$ in the case where $\Delta b^{\prime k}=2$ and 1 . The result coincides with ref. [8].

Note that any orbifold model lead to integer values of $\Delta b^{\prime k}$, although the elements of the modular weights are fractional. Types shown explicitly in Tables 6, 7, 8-1 and 8-2 are available for model building. In the above analysis, we have considered the case of the SUSY-breaking at $M_{Z}$. We can easily extend to other cases, e.g., the SUSY-breaking at $1 \mathrm{TeV}$.

\section{Hidden Sector}


It is expected that the hidden sector plays a role in the SUSY-breaking. The gaugino condensation in the hidden sector is one of the most attractive proposals to break the SUSY. In this scenario, the condensation around $10^{13} \mathrm{GeV}$ leads to soft SUSY-breaking terms at the weak scale in the observable sector. Therefore it is very important to investigate what kinds of hidden sectors are consistent with the MSSM in the observable sector and to estimate the values of the gauge coupling constants at $10^{13} \mathrm{GeV}$ in the hidden sectors.

For concreteness, we discuss the hidden sector of the minimal string model of the $Z_{6}$-I with $\Delta b^{\prime k}=3$ shown in the second row of Table 6 . We take a $\operatorname{SU}(N)^{\prime}$ group as the hidden sector. If the hidden sector has no matter field with a nontrivial representation under the $\mathrm{SU}(N)^{\prime}$ group, we obtain $b^{i}=-N$. This hidden sector is not allowed, because $b_{3}^{\prime j}=-7 / 2(j=1,2)$. Thus we need some hidden matter fields with non-trivial representations. Especially for $\operatorname{SU}(N)^{\prime}(N>4)$, we need matter fields with the modular weights to increase $b_{\text {hid }}^{j}$, i.e., $n^{i}=(0,0,-1)$, which corresponds to the untwisted sector associated with the third plane. The other modular weights decrease the value of $b_{\text {hid }}^{j}$. Here we restrict ourselves to matter fields with $N$-dim fundamental representations. For $b_{\text {hid }}^{\prime j} \geq-7 / 2$, we need the $M(M \geq 2 N-7)$ matter fields in the above untwisted sector.

For example, an $\mathrm{SU}(9)^{\prime}$ hidden gauge group must have at least eleven matter fields with the 9-dim representation in the untwisted sector. However such a larger number of the matter fields seems to be unrealistic. Actually in the case with vanishing Wilson lines the untwisted matter spectra are shown in ref. 25] and those spectra do not include such a large number of the matter fields with 9-dim representation. If the Wilson lines do not change the gauge group, we can easily estimate the number of the matter fields in the case of non-vanishing Wilson lines. The presence of the Wilson lines restricts the matter fields. Therefore the number of the matter fields without Wilson lines is the maximum among the general cases. Further we have to consider the case that the Wilson lines break gauge groups. Namely we can derive the SU(9)' group by the Wilson lines from a large group. Even including these cases, we cannot find eleven or more matter fields, but at most only the three matter fields are allowed. Similarly we cannot obtain nine (seven) or more matter fields with the 8-dim (7-dim) representation of the $\mathrm{SU}(8)^{\prime}\left(\mathrm{SU}(7)^{\prime}\right)$ group. Thus the minimal string model from the $Z_{6}$-I with $\Delta b^{\prime k}=3$ cannot have $\mathrm{SU}(N)^{\prime}(N>6)$ hidden gauge groups. On the other hand, we have the possibilty to obtain the required number of the matter fields for the 
other $\mathrm{SU}(N)^{\prime}$ hidden groups. The first and fourth columns of Table 9 show the smallest numbers of the matter fields in the hidden sectors satisfying the duality anomaly cancellation condition with $b_{3}^{\prime j}=-7 / 2$. In the column $(N, M)$ shows the $M$ matter fields with the $N$-dim representation under the $\mathrm{SU}(N)^{\prime}$ hidden group.

Next we estimate the gauge coupling constants of the hidden groups allowed by the above arguments. We eliminate $\delta_{\mathrm{GS}}^{k}$ and $T_{k}$ in eq.(3.10) for the hidden gauge couplings using the $\mathrm{SU}(2)$ and $\mathrm{SU}(3)$ gauge couplings. Then we obtain the following equation [20],

$$
\alpha_{\text {hid }}^{-1}(\mu)=\alpha_{X}^{-1}+\frac{1}{4 \pi} b_{\text {hid }} \log \frac{M_{X}^{2}}{\mu^{2}}+\frac{1}{4 \pi}\left(b_{\text {hid }}-1+4 \frac{b_{\text {hid }}^{\prime k}-b_{2}^{\prime k}}{\Delta b^{\prime k}}\right) \log \frac{M_{\text {st }}^{2}}{M_{X}^{2}},
$$

where $b_{\text {hid }}$ is an $\mathrm{N}=1 \beta$-function coefficient for the hidden group, i.e., $b_{\text {hid }}=$ $-3 N+M$ for the $M$ matter fields in the $\mathrm{SU}(N)^{\prime}$ group. Note that $M_{X}$ means the unification scale of $\mathrm{SU}(3)$ and $\mathrm{SU}(2)$ in the observable sector, and the gauge coupling of the hidden sector does not always unify with the $\mathrm{SU}(3)$ and $\mathrm{SU}(2)$ gauge couplings at $M_{X}$. We use $\alpha_{X}^{-1}=24.5$ at $M_{X}=10^{16.2} \mathrm{GeV}$ to estimate $\alpha_{\text {hid }}^{-1}$ at $10^{13.0} \mathrm{GeV}$. The results are listed in the second and fifth columns of Table 9 , while the third and sixth columns show blow-up scales $\Lambda(\mathrm{GeV})$ where $\alpha_{\text {hid }}^{-1}(\Lambda)=$ 0 .

Similarly we can find the hidden sector satisfying the duality anomaly cancellation condition with the minimal string model in the case of the $Z_{8}$-I orbifold. For simplicity we consider here some smaller numbers of the matter fields in the hidden sector. Tables 10-1, 10-2 and 10-3 show only allowed hidden sectors where the numbers of the matter fields are less than 3, 6 and 11 for $\mathrm{SU}(2)^{\prime}, \mathrm{SU}(3)^{\prime}$ and $\mathrm{SU}(N)^{\prime}(N>3)$, respectively. For the case with the three matter fields in $\operatorname{SU}(2)^{\prime}$, the gauge coupling constant does not blow up at higher than $100 \mathrm{GeV}$. The larger hidden group requires the larger number of the matter fields. The second column of Tables 10-1, 10-2 and 10-3 shows the least number of the matter fields in a gauge group for each type of minimal string model with $\Delta b^{\prime k}=4$ found in Table 7 , and the number in the parentheses corresponds to the case with $\Delta b^{\prime k}=3$. For example, Type 3 with $\Delta b^{\prime k}=4$ is allowed to have the $M(M \geq 3)$ matter fields with the 4-dim representations of the hidden $\mathrm{SU}(4)^{\prime}$ group. Similarly the least numbers of the $N$-dim hidden matter fields for Type 3 are 5,7 and 9 for the $\mathrm{SU}(5)^{\prime}$, SU(6)' and $\mathrm{SU}(7)^{\prime}$ hidden groups, respectively. Type 3 does not allow the ten or less matter fields with the 8-dim representation of the $\mathrm{SU}(8)^{\prime}$ group. The hidden sectors are constrained much more severely than the case of ref. [20], 
where is studied the duality anomaly cancellation condition for the observable $\mathrm{SU}(3), \mathrm{SU}(2)$ and hidden groups. Note that it is difficult for some types to have the hidden sectors such as considered here. Especially Types 2, 5 and 9 15 with $\Delta b^{\prime k}=3$ cannot have hidden sectors with $M(M<11)$ matter fields under $\mathrm{SU}(N)^{\prime}(N>3)$.

In addition to $n^{i}=(0,0,-1)$, the $Z_{8}$-I and $Z_{6}$-II orbifold models have the modular weights increasing the values of $b_{\text {hid }}^{\prime j}$, which correspond to the twisted sectors. Therefore we do not have a constraint from the largest number of the untwisted matter fields, which is used for the $Z_{6}$-I orbifold models.

Next we can estimate the gauge coupling constants of the above hidden groups. The third and fourth columns of Tables 10-1, 10-2 and 10-3 show the gauge couplings $\alpha_{\text {hid }}^{-1}$ at $10^{13.0} \mathrm{GeV}$ and the blow-up scales $\Lambda(\mathrm{GeV})$ in the case of $\Delta b^{\prime}=4$, respectively. The numbers in the parentheses correspond to the case of $\Delta b^{\prime}=3$. Note that the maximum values of $\alpha_{\text {hid }}^{-1}\left(10^{13.0} \mathrm{GeV}\right)$ increase by 1.1 as the number of the matter fields becomes larger by one. Even in the case of the sixteen matter fields of the $\mathrm{SU}(8)^{\prime}$, gauge coupling blows up at higher than $10^{13.4} \mathrm{GeV}$.

Similarly we can find the hidden sectors and their gauge coupling constants of the $Z_{6}$-II minimal string models. Tables $11-1$ and 11-2 show less than 4, 6 and 11 matter fields with the 2-dim, 3-dim and $N$-dim representation in the $\mathrm{SU}(2)^{\prime}, \mathrm{SU}(3)^{\prime}$ and $\mathrm{SU}(N)^{\prime}(N \geq 4)$, respectively. Forbidden hidden sectors are omitted there. The second, fourth and sixth columns show the gauge coupling constants at $10^{13.0} \mathrm{GeV}$, consistent with the minimal string models which have $\Delta b^{\prime}=6,5$ and 4 , respectively. Numbers in the third, fifth and seventh columns correspond to blow-up scales $\Lambda(\mathrm{GeV})$ of the hidden gauge coupling constants. We need the thirteen matter fields with the 7-dim representation for the gauge coupling constants of $\mathrm{SU}(7)^{\prime}$ not so as to blow up at higher than $10^{13.0} \mathrm{GeV}$. Even if we consider the case with the fourteen matter fields with the fundamental representation, the gauge coupling constants of $\mathrm{SU}(8)^{\prime}$ and $\mathrm{SU}(9)^{\prime}$ blow up at higher than $10^{13.7} \mathrm{GeV}$ and $10^{14.4} \mathrm{GeV}$, respectively.

\section{Yukawa Coupling}

In this section, we study Yukawa couplings as a further phenomenological application of the above models. The orbifold models have restrictive selection 
rules for the Yukawa couplings [29, 24, 16]. A point group selection rule requires that a product of point group elements should be an identity. Sectors allowed to couple are shown explicitly in ref. 24, 16]. Further the $Z_{N}$ invariance requires a product of the $Z_{N}$ phases from the oscillated states to be zero. Thus the Yukawa couplings of the oscillated states are much more restrictive.

First of all, we apply the above selection rules to the $Z_{6}$-I minimal string model with $\Delta b^{\prime k}=3$. Then we find that the selection rules do not allow any coupling. Next we analyze the $Z_{8}$ I minimal sting models similarly. Allowed couplings of the sectors are obtained as follows,

$$
U_{1} U_{2} U_{3}, \quad T_{1} T_{2} T_{5}, \quad T_{2} T_{2} T_{4}, \quad U_{2} T_{4} T_{4}
$$

where $U_{i}$ is the untwisted sector associated with the $i$-th plane and $T_{\ell}$ represents $\theta^{\ell}$-twisted sector. The combination of the modular weights corresponding to Type 6 with $\Delta b^{\prime k}=4$ allow only the Yukawa coupling for the top quark. The other types with $\Delta b^{\prime k}=4$ do not permit any couplings. The top Yukawa coupling is allowed by a combination of $\left(U_{3}, U_{3}, T_{5}\right)$ for $Q,\left(U_{1}, U_{3}, T_{5}\right)$ for $U,\left(U_{2}, \tilde{T}_{1}\left(N_{1}=\right.\right.$ $\left.2 / 8), \tilde{T}_{5}\left(N_{1}=2 / 8\right), \tilde{T}_{5}\left(N_{3}=2 / 8\right), \tilde{T}_{5}\left(N_{3}=2 / 8\right),\right)$ for $L$ and $H$, and $\left(T_{2}, T_{5}, T_{5}\right)$ for $E$, where $\tilde{T}_{\ell}\left(N_{i}\right)$ indicates the $\theta^{\ell}$-twisted sector with the oscillation number $N_{i}$ corresponding to the $i$-th plane. Note that the hidden sectors of Type 6 are very constrained.

We can investigate the Yukawa couplings of the $Z_{8}$-I minimal string models with $\Delta b^{\prime k}=3$ in the similar way. Types $4,11,13$ and 14 permit a Yukawa coupling for the top quark alone, and Type 9 permit Yukawa couplings for the top and bottom quarks. The others do not allow any coupling. We remark that among types allowing the couplings only Type 4 appears in Tables 10-1, 10-2 and 10-3, i.e., only Type 4 is possible to have a rich structure in the hidden sector.

Similarly we study the Yukawa couplings of the $Z_{6}$-II minimal string models, where we have the following couplings,

$$
U_{1} U_{2} U_{3}, \quad T_{1} T_{2} T_{3}, \quad T_{1} T_{1} T_{4}, \quad T_{2} T_{4} U_{3}, \quad T_{3} T_{3} U_{2}
$$

For $\Delta b^{\prime k} \geq 5$, Type 3, 4 and 8 allow only the top Yukawa coupling and the other types permit no coupling. For $\Delta b^{\prime k}=4$, most of types allow only the top Yukawa coupling but Type 12 allows the top and bottom Yukawa couplings. In Type 12 three generations of the quark doublets, $Q$ are assigned to $\left(U_{1-3}, U_{1-3}, T_{3}\right)$, where $U_{1-3}$ denotes $U_{1}$ or $U_{3}$. Further $U, D$ and $E$ belong to $\left(T_{2}, T_{3}, \tilde{T}_{4}\left(N_{2}=2 / 6\right)\right)$, 
$\left(T_{2}, \tilde{T}_{2}\left(N_{1}=2 / 6\right), \tilde{T}_{4}\left(N_{2}=2 / 6\right)\right)$ and $\left(U_{2}, U_{2}, T_{2}\right)$, respectively. Anomg $L$ and $H$, three matter fields belong to $T_{4}$ and the others belong to $T_{1}$, and further three of the five have oscillation number $N_{1}=2 / 6$.

In the above models the Yukawa couplings are fairly constrained. However, we remark that nonrenormalizable coupling could also lead other Yukawa couplings. These couplings are considered to be suppressed by at most $1 / M_{\mathrm{st}}$.

\section{Conclusion and Discussion}

In this paper we have studied the possibility of obtaining the MSSM with the measured values of the gauge coupling constants from the $Z_{N}$ orbifold models. We have used the duality anomaly cancellation condition and the moduli dependent threshold corrections to the gauge coupling constants. We have restricted ourselves to the case where the expectation value of the moduli field is of order one. Under the restriction, the $Z_{8}$-I and $Z_{6}$-II orbifold models are very promising. Allowed combinations of the modular weights are shown in Tables 6, 7, 8-1 and 8-2. That is very useful for model building. We have also found that the minimal string models on the $Z_{6}$-I, $Z_{6}$-II and $Z_{8}$-I orbifolds are possible to have the levels of $k_{1}=29 / 21,1 \leq k_{1} \leq 32 / 21$ and $1 \leq k_{1} \leq 41 / 21$, respectively, for $\Delta b^{\prime k} \geq 3$. Although the GUT prediction value $k_{1}=5 / 3$ is included only in $Z_{8}$-I, it is also included in the $Z_{6}$-II as discussed in ref. [B] if $\Delta b^{\prime k}=2$ or 1 is permitted. Further we have the lower bounds for the values of $T_{k}$ as $T_{k} \geq 11,6.5$ and 8.8 in the minimal string models derived from $Z_{6}$-I,$Z_{6}$-II and $Z_{8}$-I orbifold constructions, respectively. Note that any orbifold model always derive integer values of $\Delta b^{\prime k}$ in spite of fractional values of the modular weights.

In this paper we have assumed that the soft SUSY-breaking masses are universal and of order $M_{Z}$. However the string theories in general derive non-universal soft masses [8, 30]. Ref. [31] shows that the non-universality of the soft masses is important for analyzing the gauge coupling unification. The non-universality often increases the unification scale $M_{X}$ and makes it possible that smaller threshold corrections could explain the measured values of the gauge coupling constants. In this non-universal cases the smaller values of $\Delta b^{\prime k}$, e.g. $\Delta b^{\prime k}=1$ or 2 could lead to $T$ of order one. Therefore it is very intriguing to analyze similarly as the above with including the non-universality of the soft SUSY-breaking masses. 
We have also studied the hidden sectors of the minimal string models. The structures of the hidden sectors are also strongly constrained by the duality anomaly cancellation condition. Actually, hidden sectors with smaller number of the matter fields are often ruled out. We have restricted ourselves to the case of the hidden sectors with the $\mathrm{SU}(N)^{\prime}$ gauge groups. We can easily extend other hidden gauge groups like $\mathrm{SO}(2 N)^{\prime}$ and $\mathrm{E}_{N}^{\prime}$.

At last we have discussed the Yukawa couplings allowed in the above minimal string models. The condition of the Yukawa couplings can be used as a phenomenological constraint for the minimal string models. The constraint on the Yukawa couplings is discussed for the $Z_{N} \times Z_{M}$ orbifold models in ref. 32.

Although we have not investigated $Z_{12}$-I and $Z_{12}$-II orbifold models, the above procedure can be also applied to them. One will be able also to investigate the supersymmetric standard models with some extensions by extra matters 33 through the similar estimations, although singlets like the right-handed neutrinos have been included in the above discussions. Further string models could be expected to have some extra U(1)'s in general. Thus it is interesting to extend the above analyses including models with extra U(1)'s. Inclusion of the extra U(1)'s leads another constraint due to the duality anomaly cancellation condition.

\section{Acknowledgement}

The authors would like to thank D. Suematsu and Y. Yamagishi for useful discussions. The work of T.K. is supported in part by Soryuushi Shogakukai. 


\section{References}

[1] V.S. Kaplunovsky, Nucl. Phys. B307 (1988) 145.

[2] L. Dixon, J. Harvey, C. Vafa and E. Witten, Nucl. Phys. B261 (1985) 678; Nucl. Phys. B274 (1986) 285.

L.E. Ibáñez, J. Mas, H.P. Nilles and F. Quevedo, Nucl. Phys. B301 (1988) 157.

[3] L.E. Ibáñez, J.E. Kim, H.P. Nilles and F. Quevedo, Phys.Lett. B191 (1987) 282.

A. Font, L.E. Ibáñez, H.-P. Nilles and F. Quevedo, Phys. Lett. B210 (1988) 101.

J.A Casas and C. Muñoz, Phys. Lett. B209 (1988) 214; Phys. Lett. B212 (1988) 343; Phys. Lett. B214 (1988) 63.

Y. Katsuki, Y. Kawamura, T. Kobayashi, N. Ohtsubo, Y. Ono and K. Tanioka, Nucl. Phys. B341 (1990) 611.

J.A. Casas, A. de la Macorra, M. Mondragón and C. Muñoz, Phys. Lett. B247 (1990) 50.

H. Kawabe, T. Kobayashi and N. Ohtsubo, Phys. Lett. B322 (1994) 331.

[4] J. Ellis, S. Kelley and D.V. Nanopoulous, Phys. Lett. B260 (1991) 131.

U. Amaldi, W. de Boer and H. Fürstenau, Phys. Lett. B260 (1991) 447.

P. Langacker and M. Luo, Phys. Rev. D44 (1991) 817.

G.G. Ross and R.G. Roberts, Nucl. Phys. B377 (1992) 571.

[5] L.J. Dixon, V.S. Kaplunovsky and J. Louis, Nucl. Phys. B355 (1991) 649.

I. Antoniadis, K.S. Narain and T.R. Taylor, Phys. Lett. B267 (1991) 37.

[6] J.-P. Derendinger, S. Ferrara, C. Kounnas and F. Zwirner, Nucl. Phys. B372 (1992) 145.

[7] L. E. Ibáñez, D. Lüst and G. G. Ross, Phys. Lett. B272 (1991) 251.

[8] L.E. Ibáñez and D. Lüst, Nucl. Phys. B382 (1992) 305.

[9] L.E. Ibáñez, Phys. Lett. B318 (1993) 73. 
[10] H. Kawabe, T. Kobayashi and N. Ohtsubo, Phys. Lett. B325 (1994) 77.

[11] K. Kikkawa and M. Yamasaki, Phys. Lett. B149 (1984) 357.

N. Sakai and I. Senda, Prog. Theor. Phys. 75 (1986) 692.

[12] M.B. Green and J.H. Schwarz, Phys. Lett. B149 (1984) 117.

[13] J.-P. Derendinger, S. Ferrara, C. Kounnas and F. Zwirner, Phys. Lett. B271 (1991) 307.

[14] L.E. Ibáñez, H.P. Nilles and F. Quevedo, Phys. Lett. B187 (1987) 25.

[15] T. Kobayashi and N. Ohtsubo, Phys. Lett. B257 (1991) 56.

[16] T. Kobayashi and N. Ohtsubo, Int.J. Mod. Phys. A9 (1994) 87.

[17] H.P. Nilles, Phys. Lett. B115 (1982) 193.

S. Ferrara, L. Girardello and H.P. Nilles, Phys. Lett. B125 (1983) 457.

J.P. Derendinger, L.E. Ibáñez and H.P. Nilles, Phys. Lett. B155 (1985) 65.

M. Dine, R. Rohm N. Seiberg and E. Witten, Phys. Lett. B156 (1985) 55.

C. Kounnas and M. Porrati, Phys. Lett. B191 (1987) 91.

J.A. Casas, Z. Lalak, C. Muñoz and G.G. Ross, Nucl. Phys. B347 (1990) 243.

[18] A. Font, L.E. Ibáñez, D. Lüst and F. Quevedo, Phys. Lett. B245 (1990) 401.

S. Ferrara, N. Magnoli, T.R. Taylor and G. Veneziano, Phys. Lett. B245 (1990) 409.

M. Cvetič, A. Font, L.E. Ibáñez, D. Lüst and F. Quevedo, Nucl. Phys. B361 (1991) 194.

B. de Carlos, J.A. Casas and C. Muñoz, Nucl. Phys. B399 (1993) 623.

[19] A. de la Macorra and G. G. Ross, Nucl. Phys. B404 (1993) 321; Phys. Lett. B325 (1994) 85.

[20] T. Kobayashi, Phys. Lett. B326 (1994) 231. 
[21] D. Lewellen, Nucl. Phys. B337 (1990) 61.

A. Font, L.E. Ibáñez and F. Quevedo, Nucl. Phys. B345 (1990) 389.

[22] S. Ferrara, D. Lüst, A. Shapere and S. Theisen, Phys. Lett. B225 (1989) 363.

S. Ferrara, D. Lüst and S. Theisen, Phys. Lett. B233 (1989) 147.

[23] L.J. Dixon, V.S. Kaplunovsky and J. Louis, Nucl. Phys. B329 (1990) 27.

[24] T. Kobayashi and N. Ohtsubo, Phys. Lett. B245 (1990) 441.

[25] Y. Katsuki, Y. Kawamura, T. Kobayashi, N. Ohtsubo, Y. Ono and K. Tanioka, Phys. Lett. B218 (1989) 169.

[26] Y. Katsuki, Y. Kawamura, T. Kobayashi, N. Ohtsubo and K. Tanioka, Prog. Theor. Phys. 82 (1989) 171.

Y. Katsuki, Y. Kawamura, T. Kobayashi, N. Ohtsubo, Y. Ono and K. Tanioka, Phys. Lett. B227 (1989) 381.

[27] P. Mayr and S. Stieberger, Preprint MPI-Ph/93-07, TUM-TH-152/93 hepth/9303017).

D. Bailin, A.Love, W.A. Sabra and S. Thomas, Preprint QMW-TH-93/22, SUSX-TH-93/14 (hep-th/9310008).

[28] M. Spalinski, Phys. Lett. B275 (1992) 47.

J. Erler, D. Jungnickel and H.P. Nilles, Phys. Lett. 276 (1992) 303.

D. Bailin, A.Love, W.A. Sabra and S. Thomas, Preprint SUSX-TH-93/17 (hep-th/9312122).

[29] L. Dixon, D. Friedan, E. Martinec and S. Shenker, Ncul. Phys. B282 (1987) 13.

Hamidy and C. Vafa, Nucl. Phys. B279 (1987) 465.

J.A Casas, F. Gomez and C. Muñoz, Int. J. Phys. A8 (1993) 455.

[30] B. de Carlos, J. A. Casas and C. Muñoz, Phys. Lett. B299 (1993) 234.

A. Brignole, L. E. Ibáñez and C. Muñoz, preprint FTUAM-26/93 (hepph/9308271). 
[31] T. Kobayashi, D. Suematsu and Y. Yamagishi, Phys. Lett. B329 (1994) 27.

[32] T. Kobayashi, preprint Kanazawa-94-10 (hep-ph/9406238), to be publishied in Int.J. Mod. Phys. A.

[33] I. Antoniadis, J. Ellis, S. Kelley and D.V. Nanopoulos, Phys. Lett. B272 (1991) 31.

A.E. Faraggi, Phys. Lett. B302 (1993) 202. 
Table 1. modular weights in twisted sectors on $Z_{4}$ orbifold

\begin{tabular}{|c||c|c|c|c|c|c|}
\hline \multirow{2}{*}{$\begin{array}{c}\text { Twisted } \\
\text { sector }\end{array}$} & $4 n^{i}$ & \multicolumn{5}{|c|}{ Lower-bound of $k_{1}$} \\
\cline { 3 - 7 } & & $(3,2)_{1 / 6}$ & $(\overline{3}, 1)_{1 / 3}$ & $(\overline{3}, 1)_{-2 / 3}$ & $(1,2)_{ \pm 1 / 2}$ & $(1,1)_{1}$ \\
\hline \hline$\theta$ & $(-3,-3,-2)$ & $4 / 15$ & $16 / 51$ & $64 / 51$ & $4 / 7$ & $16 / 11$ \\
& $(\underline{-7,-3,-2)}$ & - & $16 / 15$ & - & $4 / 3$ & - \\
\hline$\theta^{2}$ & $(-2,-2,0)$ & $1 / 6$ & $4 / 15$ & $16 / 15$ & $1 / 2$ & $4 / 3$ \\
\hline
\end{tabular}

Table 2. Modular weights in twisted sectors on $Z_{6}$-I orbifold

\begin{tabular}{|c||c|c|c|c|c|c|c|}
\hline \multirow{2}{*}{$\begin{array}{c}\text { Twisted } \\
\text { sector }\end{array}$} & $6 n^{i}$ & \multicolumn{5}{|c|}{ Lower-bound of $k_{1}$} & \multirow{2}{*}{$\mathrm{SU}(N)^{\prime}$} \\
\cline { 3 - 8 } & & $(3,2)_{1 / 6}$ & $(\overline{3}, 1)_{1 / 3}$ & $(\overline{3}, 1)_{-2 / 3}$ & $(1,2)_{ \pm 1 / 2}$ & $(1,1)_{1}$ & $\underline{N}$ \\
\hline \hline \multirow{2}{*}{$\theta$} & $(-5,-5,-2)$ & $1 / 6$ & $4 / 15$ & $16 / 15$ & $1 / 2$ & $4 / 3$ & + \\
& $(-11,-5,-2)$ & - & $4 / 9$ & $16 / 9$ & $3 / 4$ & $12 / 7$ & + \\
& $(-17,-5,-2)$ & - & $4 / 3$ & - & $3 / 2$ & - & $N \leq 6$ \\
& $(-11,-11,-2)$ & - & $4 / 3$ & - & $3 / 2$ & - & $N \leq 6$ \\
& $(-5,-5,4)$ & - & $4 / 3$ & - & $3 / 2$ & - & $N \leq 6$ \\
\hline$\theta^{2}$ & $(-4,-4,-4)$ & $1 / 3$ & $1 / 3$ & $4 / 3$ & $3 / 5$ & $3 / 2$ & + \\
\hline$\theta^{3}$ & $(-3,-3,0)$ & $1 / 6$ & $4 / 15$ & $16 / 15$ & $1 / 2$ & $4 / 3$ & + \\
\hline
\end{tabular}


Table 3. Modular weights in twisted sectors on $Z_{6}$-II orbifold

\begin{tabular}{|c|c|c|c|c|c|c|c|}
\hline \multirow{2}{*}{$\begin{array}{c}\text { Twisted } \\
\text { sector }\end{array}$} & \multirow[t]{2}{*}{$6 n^{i}$} & \multicolumn{5}{|c|}{ Lower-bound of $k_{1}$} & \multirow{2}{*}{$\begin{array}{c}\mathrm{SU}(N)^{\prime} \\
\underline{N}\end{array}$} \\
\hline & & $(3,2)_{1 / 6}$ & $(\overline{3}, 1)_{1 / 3}$ & $(\overline{3}, 1)_{-2 / 3}$ & $(1,2)_{ \pm 1 / 2}$ & $(1,1)_{1}$ & \\
\hline \multirow[t]{2}{*}{$\theta$} & $(-5,-4,-3)$ & $1 / 3$ & $4 / 13$ & $16 / 13$ & $9 / 16$ & $36 / 25$ & + \\
\hline & $(-11,-4,-3)$ & - & $4 / 7$ & - & $9 / 10$ & $36 / 19$ & + \\
\hline \multirow[t]{3}{*}{$\theta^{2}$} & $(-4,-2,0)$ & $1 / 7$ & $1 / 5$ & $4 / 5$ & $9 / 19$ & $9 / 7$ & + \\
\hline & $(-10,-2,0)$ & - & $1 / 2$ & 2 & $9 / 7$ & - & $N=2$ \\
\hline & $(-4,4,0)$ & - & $1 / 2$ & 2 & $9 / 7$ & - & $N=2$ \\
\hline$\theta^{3}$ & $(-3,0,-3)$ & $1 / 6$ & $4 / 15$ & $16 / 15$ & $1 / 2$ & $4 / 3$ & + \\
\hline \multirow[t]{3}{*}{$\theta^{4}$} & $(-2,-4,0)$ & $1 / 7$ & $1 / 5$ & $4 / 5$ & $9 / 19$ & $9 / 7$ & + \\
\hline & $(-2,-10,0)$ & - & $1 / 2$ & 2 & $9 / 7$ & - & $N=2$ \\
\hline & $(4,-4,0)$ & - & $1 / 2$ & 2 & $9 / 7$ & - & $N=2$ \\
\hline
\end{tabular}


Table 4. Modular weights in twisted sectors on $Z_{8}$-I orbifold

\begin{tabular}{|c||c|c|c|c|c|c|}
\hline \multirow{2}{*}{ Twisted } & $8 n^{i}$ & \multicolumn{5}{|c|}{ Lower-bound of $k_{1}$} \\
\cline { 3 - 7 } sector & & $(3,2)_{1 / 6}$ & $(\overline{3}, 1)_{1 / 3}$ & $(\overline{3}, 1)_{-2 / 3}$ & $(1,2)_{ \pm 1 / 2}$ & $(1,1)_{1}$ \\
\hline \hline \multirow{2}{*}{$\theta$} & $(-7,-3,-6)$ & $16 / 87$ & $64 / 231$ & $256 / 231$ & $16 / 31$ & $64 / 47$ \\
& $(-15,-3,-6)$ & $16 / 15$ & $64 / 159$ & $256 / 159$ & $16 / 23$ & $64 / 39$ \\
& $(-23,-3,-6)$ & - & $64 / 87$ & - & $16 / 15$ & - \\
& $(-7,-3,-14)$ & - & $64 / 87$ & - & $16 / 15$ & - \\
\hline$\theta^{2}$ & $(-6,-6,-4)$ & $4 / 15$ & $16 / 51$ & $64 / 51$ & $4 / 7$ & $16 / 11$ \\
& $(-14,-6,-4)$ & - & $16 / 15$ & - & $4 / 3$ & - \\
\hline$\theta^{4}$ & $(-4,-4,0)$ & $1 / 6$ & $4 / 15$ & $16 / 15$ & $1 / 2$ & $4 / 3$ \\
\hline$\theta^{5}$ & $(-3,-7,-6)$ & $16 / 87$ & $64 / 231$ & $256 / 231$ & $16 / 31$ & $64 / 47$ \\
& $(-3,-15,-6)$ & $16 / 15$ & $64 / 159$ & $256 / 159$ & $16 / 23$ & $64 / 39$ \\
& $(-3,-23,-6)$ & - & $64 / 87$ & - & $16 / 15$ & - \\
& $(-3,-7,-14)$ & - & $64 / 87$ & - & $16 / 15$ & - \\
\hline
\end{tabular}


Table 5. Modular weights in twisted sectors on $Z_{8}$-II orbifold

\begin{tabular}{|c||c|c|c|c|c|c|}
\hline \multirow{2}{*}{ Twisted } & \multirow{2}{*}{$8 n^{i}$} & \multicolumn{5}{|c|}{ Lower-bound of $k_{1}$} \\
\cline { 3 - 7 } sector & & $(3,2)_{1 / 6}$ & $(\overline{3}, 1)_{1 / 3}$ & $(\overline{3}, 1)_{-2 / 3}$ & $(1,2)_{ \pm 1 / 2}$ & $(1,1)_{1}$ \\
\hline \hline \multirow{2}{*}{$\theta$} & $(-7,-5,-4)$ & $16 / 69$ & $64 / 213$ & $256 / 213$ & $16 / 29$ & $64 / 45$ \\
& $(-15,-5,-4)$ & - & $64 / 141$ & $256 / 141$ & $16 / 21$ & $64 / 37$ \\
& $(-23,-5,-4)$ & - & $64 / 69$ & - & $16 / 13$ & - \\
\hline$\theta^{2}$ & $(-6,-2,0)$ & $4 / 33$ & $16 / 69$ & $64 / 69$ & $4 / 9$ & $16 / 13$ \\
& $(-14,-2,0)$ & - & $16 / 33$ & $64 / 33$ & $4 / 5$ & $16 / 9$ \\
& $(-6,6,0)$ & - & $16 / 33$ & $64 / 33$ & $4 / 5$ & $16 / 9$ \\
\hline$\theta^{3}$ & $(-5,-7,-4)$ & $16 / 69$ & $64 / 213$ & $256 / 213$ & $16 / 29$ & $64 / 45$ \\
& $(-5,-15,-4)$ & - & $64 / 141$ & $256 / 141$ & $16 / 21$ & $64 / 37$ \\
& $(-5,-23,-4)$ & - & $64 / 69$ & - & $16 / 13$ & - \\
\hline$\theta^{4}$ & $(-4,-4,0)$ & $1 / 6$ & $4 / 15$ & $16 / 15$ & $1 / 2$ & $4 / 3$ \\
\hline$\theta^{6}$ & $(-2,-6,0)$ & $4 / 33$ & $16 / 69$ & $64 / 69$ & $4 / 9$ & $16 / 13$ \\
& $(-2,-14,0)$ & - & $16 / 33$ & $64 / 33$ & $4 / 5$ & $16 / 9$ \\
& $(6,-6,0)$ & - & $16 / 33$ & $64 / 33$ & $4 / 5$ & $16 / 9$ \\
\hline
\end{tabular}


Table 6. Minimal String Model from $Z_{6}$-I orbifold

\begin{tabular}{|c|c|c|c|c|c|c|}
\hline$\#$ & $\Delta b^{\prime k}(T)$ & $Q$ & $U$ & $D$ & $L, H$ & $E$ \\
\hline \hline 1 & $3(11)$ & $3,3,4$ & $4,4,6$ & $9,9,9$ & $6,7,7,8,8$ & $3,3,4$ \\
2 & $2(15)$ & $1,2,3$ & $5,6,6$ & $9,9,9$ & $3,7,7,8,8$ & $3,4,4$ \\
3 & $2(15)$ & $3,5,5$ & $5,6,6$ & $9,9,9$ & $3,7,7,8,8$ & $3,4,4$ \\
4 & $1(28)$ & $1,2,3$ & $4,4,4$ & $4,9,9$ & $6,7,7,8,8$ & $3,3,4$ \\
5 & $1(28)$ & $3,5,5$ & $4,4,4$ & $4,9,9$ & $6,7,7,8,8$ & $3,3,4$ \\
\hline
\end{tabular}


Table 7. Minimal String Model from $Z_{8}$-I orbifold

\begin{tabular}{|c|c|c|c||c|c|c|c|}
\hline \multicolumn{5}{|c||}{$\Delta b^{\prime k}=4$} & \multicolumn{4}{c|}{$\Delta b^{\prime k}=3$} \\
\hline Type & $k_{1}$ & $\left(8 b_{3}^{\prime 1}, 8 b_{3}^{\prime 2}\right)$ & $8 b_{3}^{\prime 3}$ & Type & $k_{1}$ & $\left(8 b_{3}^{\prime 1}, 8 b_{3}^{\prime 2}\right)$ & $8 b_{3}^{\prime k}$ \\
\hline \hline 1 & 1 & $(-12,-12)$ & -16 & 1 & $15 / 16$ & $(-28,-28)$ & -40 \\
2 & $23 / 21$ & $(-22,-22)$ & -12 & 2 & $7 / 6$ & $(-28,-12)$ & -32 \\
3 & $7 / 6$ & $(-36,-20)$ & -32 & 3 & $7 / 6$ & $(4,-44)$ & -32 \\
4 & $7 / 6$ & $(-4,-52)$ & -32 & 4 & $19 / 15$ & $(-28,-28)$ & -40 \\
5 & $53 / 39$ & $(-42,-42)$ & -28 & 5 & $4 / 3$ & $(-20,-20)$ & -16 \\
6 & $29 / 21$ & $(-1,-29)$ & -34 & 6 & $4 / 3$ & $(4,-44)$ & -16 \\
7 & $5 / 3$ & $(-55,-11)$ & -38 & 7 & $4 / 3$ & $(-4,-52)$ & -48 \\
8 & $5 / 3$ & $(-19,-47)$ & -38 & 8 & $53 / 39$ & $(-34,-34)$ & -28 \\
9 & $5 / 3$ & $(-51,-15)$ & -38 & 9 & $29 / 21$ & $(-14,-14)$ & -28 \\
10 & $5 / 3$ & $(-27,-39)$ & -38 & 10 & $29 / 21$ & $(-23,-51)$ & -38 \\
11 & $5 / 3$ & $(-59,-7)$ & -38 & 11 & $29 / 21$ & $(-22,-22)$ & -36 \\
12 & $5 / 3$ & $(-23,-43)$ & -38 & 12 & $17 / 12$ & $(-40,-8)$ & -48 \\
13 & $41 / 21$ & $(-23,5)$ & -46 & 13 & $17 / 33$ & $(-34,-34)$ & -12 \\
& & & 14 & $17 / 9$ & $(1,-35)$ & -38 \\
& & & 15 & $41 / 21$ & $(-43,-15)$ & 2 \\
\hline
\end{tabular}


Table 8-1. Minimal String Model from $Z_{6}$-II orbifold with $\Delta b^{\prime k}=6$ and 5

Typle 1 has $\Delta b^{\prime k}=6$ and the other correspond to $\Delta b^{\prime k}=5$ and $T=7.4$

\begin{tabular}{|c|c|c|c|c|}
\hline Type & $k_{1}$ & $6 b_{3}^{\prime 1}$ & $6 b_{3}^{\prime k}$ & $18 b_{1}^{\prime k}$ \\
\hline \hline 1 & $7 / 6$ & 8 & 4 & 248 \\
\hline 2 & $7 / 6$ & 8 & -2 & $176,200,212$ \\
3 & & 8 & 4 & $194,230,266,302$ \\
4 & & 16 & -4 & 178 \\
\hline 5 & $11 / 9$ & 9 & -3 & 135 \\
\hline 6 & $26 / 21$ & 14 & -8 & 80 \\
\hline 7 & $19 / 15$ & 5 & 1 & $77,113,149$ \\
\hline 8 & $31 / 24$ & 16 & -4 & 64,100 \\
\hline 9 & $67 / 51$ & 17 & -11 & 5,29 \\
\hline 10 & $4 / 3$ & 6 & 0 & 36 \\
11 & & 12 & -6 & 12 \\
\hline 12 & $41 / 30$ & 20 & -14 & $-82,-46$ \\
\hline 13 & $29 / 21$ & 14 & -8 & -58 \\
\hline
\end{tabular}


Table 8-2. Minimal String Model from $Z_{6}$-II orbifold with $\Delta b^{\prime k}=4$

\begin{tabular}{|c|c|c|c||c|c|c|c|}
\hline Type & $k_{1}$ & $6 b_{3}^{\prime \prime}$ & $6 b_{3}^{\prime k}$ & Type & $k_{1}$ & $6 b_{3}^{\prime \prime}$ & $6 b_{3}^{\prime k}$ \\
\hline \hline 1 & $16 / 15$ & 10 & -4 & 15 & $31 / 24$ & 16 & -10 \\
2 & $23 / 21$ & 7 & -1 & 16 & $43 / 33$ & 11 & $-11,-5$ \\
3 & $37 / 33$ & 11 & -5 & 17 & & 22 & -16 \\
4 & $7 / 6$ & 4 & $-10,2$ & 18 & $67 / 51$ & 17 & $-17,-11$ \\
5 & & 8 & $-8,-2,4$ & 19 & $4 / 3$ & 6 & $-6,0$ \\
6 & & 16 & $-10,-4$ & 20 & & 12 & $-12,-6$ \\
7 & $61 / 51$ & 17 & -11 & 21 & & 18 & -12 \\
8 & $47 / 39$ & 13 & -7 & 22 & $77 / 57$ & 19 & -13 \\
9 & $11 / 9$ & 9 & $-9,-3$ & 23 & $53 / 39$ & 13 & -7 \\
10 & $26 / 21$ & 14 & $-14,-8$ & 24 & $41 / 30$ & 20 & $-20,-14$ \\
11 & $71 / 57$ & 19 & -13 & 25 & $29 / 21$ & 7 & -19 \\
12 & $19 / 15$ & 5 & $-17,-5,1$ & 26 & & 14 & $-14,-8$ \\
13 & & 10 & $-5,-4$ & 27 & $46 / 33$ & 22 & -16 \\
14 & & 20 & -14 & 28 & $17 / 12$ & 8 & $-8,-2$ \\
\hline
\end{tabular}


Table 9. Hidden gauge coupling in $Z_{6}$-I orbifold

\begin{tabular}{|c|c|c||c|c|c|}
\hline $\mathrm{N}, \mathrm{M}$ & $\alpha_{\text {hid }}^{-1}$ & $\log _{10} \Lambda$ & $\mathrm{N}, \mathrm{M}$ & $\alpha_{\text {hid }}^{-1}$ & $\log _{10} \Lambda$ \\
\hline \hline 2,2 & $13.7-14.3$ & $5.5-5.2$ & 5,3 & 3.05 & 12.4 \\
2,3 & $14.2-14.9$ & $4.4-4.0$ & 5,4 & 3.56 & 12.3 \\
3,2 & $8.66-9.97$ & $10.1-9.8$ & 5,5 & 4.07 & 12.1 \\
3,3 & $9.17-11.1$ & $9.7-9.0$ & 5,6 & $4.58-5.89$ & $12.0-11.7$ \\
4,1 & 5.09 & 11.8 & 5,7 & $5.09-6.40$ & $11.8-11.4$ \\
4,2 & 5.60 & 11.6 & 6,5 & 1.01 & 12.8 \\
4,3 & 6.11 & 11.4 & 6,6 & 1.52 & 12.8 \\
4,4 & $6.62-7.93$ & $11.2-10.8$ & 6,7 & 2.03 & 12.6 \\
4,5 & $7.13-8.44$ & $11.0-10.6$ & 6,8 & $2.54-3.85$ & $12.5-12.3$ \\
& & & 6,9 & $3.05-4.36$ & $12.4-12.1$ \\
\hline
\end{tabular}


Table 10-1. Hidden gauge coupling in $Z_{8}$-I orbifold

\begin{tabular}{|c|c|c|c|}
\hline$N, M$ & Type & $\alpha_{\text {hid }}^{-1}$ & $\log _{10} \Lambda$ \\
\hline \hline 2,1 & 1 & 16.5 & 4.8 \\
2,2 & $2,3,5,9,12(8)$ & $17.6-19.1(19.9)$ & $2.6-3.4(2.1)$ \\
\hline 3,1 & 12 & 12.5 & 9.0 \\
3,2 & $2,5,8,10(1,4,8)$ & $11.6-13.6(12.1-14.2)$ & $8.4-9.1(8.1-8.9)$ \\
3,3 & $1,3,9$ & $11.2-14.7(12.7-15.4)$ & $7.7-8.9(7.4-8.3)$ \\
3,4 & $4,6,7,11(7)$ & $13.3-15.7(12.9-16.6)$ & $6.9-8.2(6.5-8.0)$ \\
3,5 & $(6)$ & $12.4-16.8(13.8-17.7)$ & $5.9-7.8(5.6-7.2)$ \\
\hline 4,1 & $(8)$ & $(8.0)$ & $(11.1)$ \\
4,2 & 5,10 & $7.1-8.1(7.2-9.2)$ & $11.0-11.3(10.7-11.2)$ \\
4,3 & 3,12 & $8.2-9.1(8.4-10.4)$ & $10.6-10.9(10.3-10.8)$ \\
4,4 & $2,8(1,4)$ & $7.3-10.2(8.8-11.5)$ & $10.2-11.0(9.9-10.6)$ \\
4,5 & 1,9 & $6.9-11.3(9.4-12.7)$ & $9.8-11.3(9.4-10.3)$ \\
4,6 & 7 & $7.9-12.4(8.6-13.9)$ & $9.2-10.7(8.8-10.4)$ \\
4,7 & $4,11(7)$ & $8.0-13.5(7.8-15.1)$ & $8.7-10.4(8.2-10.5)$ \\
4,8 & & $8.6-14.6(9.0-16.2)$ & $8.0-10.1(7.5-10.0)$ \\
4,9 & $(3,6,8)$ & $8.2-15.7(7.5-18.0)$ & $7.3-10.1(6.5-10.3)$ \\
4,10 & 6,13 & $7.8-16.7(10.0-18.7)$ & $6.5-10.0(5.7-9.1)$ \\
\hline
\end{tabular}


Table 10-2. Hidden gauge coupling in $Z_{8}$-I orbifold

\begin{tabular}{|c|c|c|c|}
\hline$N, M$ & Type & $\alpha_{\text {hid }}^{-1}$ & $\log _{10} \Lambda$ \\
\hline \hline 5,1 & 5 & 1.0 & 12.8 \\
5,2 & & $0.6-2.0$ & $12.6-12.9$ \\
5,3 & $(1,4,8)$ & $1.6-3.1(3.4-3.6)$ & $12.4-12.7(12.3)$ \\
5,4 & 10 & $2.7-4.2(2.7-4.5)$ & $12.1-12.4(12.1-12.5)$ \\
5,5 & 3,12 & $2.8-5.3(3.7-5.7)$ & $11.8-12.4(11.8-12.2)$ \\
5,6 & 2,8 & $2.4-6.4(4.9-9.0)$ & $11.6-12.5(11.0-11.9)$ \\
5,7 & 1,9 & $2.0-7.5(4.7-9.6)$ & $11.2-12.5(10.7-11.9)$ \\
5,8 & 7 & $3.1-8.6(3.9-10.6)$ & $10.9-12.2(10.3-12.0)$ \\
5,9 & $4,11(7)$ & $3.7-9.6(3.1-11.7)$ & $10.5-12.0(10.0-12.2)$ \\
5,10 & & $4.3-10.7(4.3-13.6)$ & $10.1-11.8(9.3-11.8)$ \\
\hline 6,3 & 5 & - & 13.6 \\
6,4 & & $-(0.0-1.1)$ & $13.2-13.5(12.8-13.2)$ \\
6,5 & $(1,4,8)$ & $-(0.0-1.7)$ & $13.0-13.3(12.6-13.2)$ \\
6,6 & 10 & $-(0.0-2.4)$ & $12.8-13.3(12.6-13.2)$ \\
6,7 & 3,12 & $0.0-1.0(0.2)$ & $12.6-13.4(11.7-13.0)$ \\
6,8 & 2,8 & $0.0-2.0(0.2-6.5)$ & $12.4-13.5(11.7-13.0)$ \\
6,9 & 1,9 & $0.0-3.1(0.1-7.1)$ & $12.1-13.3(11.3-13.2)$ \\
6,10 & 7 & $0.0-4.2(0.0-8.2)$ & \\
\hline
\end{tabular}


Table 10-3. Hidden gauge coupling in $Z_{8}$-I orbifold

\begin{tabular}{|c|c|c|c|}
\hline$N, M$ & Type & $\alpha_{\text {hid }}^{-1}$ & $\log _{10} \Lambda$ \\
\hline \hline 7,5 & 5 & - & 14.1 \\
7,6 & & - & $14.0-14.2$ \\
7,7 & $(1,4,8)$ & $-(-)$ & $13.9-14.1(13.2-13.9)$ \\
7,8 & 10 & $-(-)$ & $13.7-14.0(13.1-14.1)$ \\
7,9 & 3,12 & $-(-)$ & $13.6-14.0(13.0-13.9)$ \\
7,10 & 2,8 & $0.0-4.2(0.0-4.0)$ & $13.4-14.2(12.3-13.8)$ \\
\hline 8,7 & 5 & - & 14.6 \\
8,8 & & - & $14.5-14.7$ \\
8,9 & $(1,4,8)$ & $-(-)$ & $14.4-14.6(13.5-14.5)$ \\
8,10 & 10 & $-(-)$ & $14.3-14.5(13.5-14.7)$ \\
\hline
\end{tabular}


Table 11-1. Hidden gauge coupling in $Z_{6}$-II orbifold

\begin{tabular}{|c|c|c|c|c|c|c|}
\hline \multirow[t]{2}{*}{$N, M$} & \multicolumn{2}{|c|}{$\Delta b^{\prime}=6$} & \multicolumn{2}{|c|}{$\Delta b^{\prime}=5$} & \multicolumn{2}{|c|}{$\Delta b^{\prime}=4$} \\
\hline & $\alpha_{\text {hid }}^{-1}$ & $\log _{10} \Lambda$ & $\alpha_{\text {hid }}^{-1}$ & $\log _{10} \Lambda$ & $\alpha_{\text {hid }}^{-1}$ & $\log _{10} \Lambda$ \\
\hline 2,3 & 17.3 & 2.5 & $17.1-17.5$ & $2.4-2.7$ & $16.7-18.7$ & $1.7-2.9$ \\
\hline 3,4 & - & - & 12.3 & 8.2 & $11.3-12.8$ & $8.0-8.6$ \\
\hline 3,5 & $13.0-13.3$ & $7.4-7.6$ & $12.5-14.1$ & $7.1-7.7$ & $11.9-14.9$ & $6.8-8.0$ \\
\hline 4,4 & - & - & - & - & $5.8-6.8$ & $11.2-11.4$ \\
\hline 4,5 & - & - & $7.1-7.5$ & $10.9-11.0$ & $6.4-8.3$ & $10.6-11.1$ \\
\hline 4,6 & $8.3-8.9$ & $10.3-10.5$ & $7.7-8.9$ & $10.3-10.7$ & $7.0-9.4$ & $10.1-10.9$ \\
\hline 4,7 & $9.3-9.9$ & $8.9-9.8$ & $8.4-10.8$ & $9.6-10.3$ & $7.5-11.5$ & $9.3-10.6$ \\
\hline 4,8 & $9.3-11.9$ & 8.9-9.8 & $8.6-12.2$ & $8.8-10.1$ & $7.6-12.6$ & $8.7-10.4$ \\
\hline 4,9 & $10.3-12.9$ & $8.3-9.3$ & $9.3-13.2$ & $8.2-9.6$ & $8.2-15.2$ & $7.5-10.0$ \\
\hline 4,10 & $10.6-14.3$ & $7.4-8.7$ & $9.9-15.1$ & $7.1-9.1$ & $8.3-16.2$ & $6.7-9.8$ \\
\hline 5,5 & - & - & 1.7 & 12.6 & $0.8-2.3$ & $12.5-12.8$ \\
\hline 5,6 & 2.6 & 12.3 & $2.3-3.1$ & $12.3-12.5$ & $1.4-3.4$ & $12.2-12.7$ \\
\hline 5,7 & 3.9 & 12.1 & $3.0-4.2$ & $12.0-12.3$ & $2.0-5.0$ & $11.8-12.5$ \\
\hline 5,8 & $3.9-5.6$ & $11.6-12.0$ & $3.2-5.6$ & $11.6-12.2$ & $2.1-6.1$ & $11.5-12.5$ \\
\hline 5,9 & $4.9-6.6$ & $11.3-11.7$ & $3.8-7.4$ & $11.1-12.0$ & $2.7-8.2$ & $10.9-12.3$ \\
\hline 5,10 & $5.3-8.6$ & $10.7-11.6$ & $4.5-8.8$ & $10.6-11.8$ & $2.8-9.2$ & $10.5-12.2$ \\
\hline
\end{tabular}


Table 11-2. Hidden gauge coupling in $Z_{6}$-II orbifold

\begin{tabular}{|c|c|c|c|c|c|c|}
\hline \multirow{2}{*}{$N, M$} & \multicolumn{2}{|c|}{$\Delta b^{\prime}=6$} & \multicolumn{2}{|c|}{$\Delta b^{\prime}=5$} & \multicolumn{2}{c|}{$\Delta b^{\prime}=4$} \\
\cline { 2 - 7 } & $\alpha_{\text {hid }}^{-1}$ & $\log _{10} \Lambda$ & $\alpha_{\text {hid }}^{-1}$ & $\log _{10} \Lambda$ & $\alpha_{\text {hid }}^{-1}$ & $\log _{10} \Lambda$ \\
\hline \hline 6,6 & - & - & - & 13.6 & - & 13.7 \\
6,7 & - & - & - & $13.3-13.5$ & - & $13.2-13.7$ \\
6,8 & - & $13.1-13.3$ & - & $13.1-13.4$ & $0.0-0.1$ & $13.0-13.7$ \\
6,9 & $0.0-0.6$ & $12.9-13.1$ & $0.0-0.8$ & $12.8-13.3$ & $0.0-1.6$ & $12.7-13.6$ \\
6,10 & $0.0-2.2$ & $12.5-13.0$ & $0.0-2.2$ & $12.5-13.2$ & $0.0-2.7$ & $12.4-13.6$ \\
7,7 & - & - & - & - & - & $14.2-14.4$ \\
7,8 & - & $14.0-14.1$ & - & $14.0-14.2$ & - & $14.0-14.4$ \\
7,9 & - & $13.9-14.0$ & - & $13.8-14.2$ & - & $13.7-14.4$ \\
7,10 & - & $13.7-13.9$ & - & $13.6-14.1$ & - & $13.6-14.4$ \\
8,8 & - & 14.7 & - & $14.7-14.8$ & - & $14.8-15.0$ \\
8,10 & - & $14.4-14.6$ & - & $14.4-14.7$ & - & $14.4-15.0$ \\
9,10 & - & $-14.9-15.0$ & - & $15.0-15.1$ & - & $15.1-15.4$ \\
\hline
\end{tabular}

